\title{
La venganza del texto: expropiación de lo explícito
}

\author{
Ana León Távora
}

\begin{abstract}
"You take the blue pill and the story ends. You wake up in your bed and you believe whatever you want to believe. You take the red pill and you stay in Wonderland and I show you how deep the rabbit-hole goes" (Morfeo en The Matrix).
\end{abstract}

Resulta evidente que al acusar al texto de cometer su propio acto de venganza se está haciendo uso de una personificación: el texto vengándose de algo o de alguien, del lector en este caso en particular, como también del propio autor. El término "texto" es tomado aquí en su vertiente más general, aunque sin olvidar el hecho incuestionable de que ante todo, un texto es una construcción artificial creada conscientemente por uno o varios individuos. Si esto es cierto, ¿cuál es el propósito de dotarlo de facultades humanas como si un texto fuera capaz de ejecutar su propio acto de venganza? ¿Qué significa "expropiación," y quién es desposeído de qué? A lo largo de este trabajo se intentará dar respuestas a estas preguntas, al mismo tiempo que se analizarán la problemática disolución de la figura del autor y la diseminación del origen.

En otros tiempos el texto debía ante todo mantener la verosimilitud. De hecho, el concepto de verosimilitud era un factor esencial a fin de presentar cualquier composición escrita de la forma más plausible. Si se presta atención a la etimología de la palabra, sin embargo, se puede observar que la idea de verosimilitud encierra en sí misma un engaño o una cierta dosis de ficción, dado que algo verosímil se aproxima a la verdad, sin ser necesariamente verdadero. Como explica Edward Said en Beginnings: "The second special condition for generating narrative fiction is that the truth-whatever that may be - can only be approached indirectly, by means of a meditation that, paradoxically, because of its falseness makes the truth truer" (90). En este sentido, el texto engaña al lector, ocultando sus mecanismos internos y los recursos que evidencian su condición como artificio, presentándose como algo directamente tomado de la realidad.

Llega un momento en la historia de la literatura, no obstante, un momento no determinado ni puntual, en que el texto se rebela e inicia un despliegue de todos sus recursos estilísticos delante del lector, rompiendo de esta forma las constricciones impuestas que lo denigran como creación artificial y haciéndose así independiente de su propia mano creadora. Si se toma este acto de rebelión como un mero acto de metadiscurso, metaliteratura o metaficción, el texto contemplándose a sí mismo, se puede llegar a afirmar que esta rebelión no es ninguna novedad y tiene lugar en diferentes episodios de la historia de la literatura así como en países muy dispares. Un ejemplo remoto de esta auto-reflexión es el célebre Libro de los Muertos egipcio, en el que se encuentran pasajes como el siguiente, donde el propio manuscrito se menciona a sí mismo: "If be known book upon earth, [and be done it] into writing upon the coffin my mouth it is, he shall come forth by day in forms of existen- 
ce all [which] he pleaseth."1 Lo mismo ocurre en España con obras como el conocido soneto de Lope de Vega donde el autor explica paso a paso cómo se forma tal estrofa, al mismo tiempo que va escribiendo su soneto: "Un soneto me manda hacer Violante, / que en mi vida me he visto en tanto aprieto; / catorce versos dicen que es soneto; / burla, burlando, van los tres delante" (1-4). Por último, cabe mencionarse el conocido Tristram Shandy de Laurence Sterne, obra que en sí misma constituye un modelo de metadiscurso, a través del cual el texto se recrea en su método de composición. El acto metadiscursivo, así entendido, puede considerarse tan antiguo como la propia escritura. Pero esta auto-reflexión no constituye el único acto de venganza que tenemos que afrontar como lectores. Algo bastante más complicado, sin duda, es el concepto de "self-reflexive metaphor" o metáfora autoreflexiva que propone la autora Katherine Hayles en su obra Chaos Bound. Hayles incluye un capítulo dedicado al estudio de este concepto, que abre con la afirmación de que "in a sense, all language is metaphoric" (31). Para Hayles cualquier manifestación lingüística, incluso en sus dimensiones más científicas, es una metáfora. La autora explica de forma más específica que el concepto de metáfora auto-reflexiva "could be imaged as a compass with one leg moving freely and the other resting not on ground, but on the leg of another compass" (33). Para ilustrar mejor esta idea, Hayles utiliza el emblema de Borges de la escalera que conduce a un espacio vacío, "not to a door, but to vertigo," aunque quizá resulte más eficaz otra imagen similar, esta vez del artista holandés M.Escher. En su pintura titulada Relativity, Escher crea un juego de perspectivas imposibles mediante el cual se forma un laberinto de escaleras que se entrelazan, cada escalera conduciendo a otra escalera y así sucesivamente. El problema de la metáfora auto-reflexiva, en este sentido, no radica simplemente en algo que se contempla a sí mismo, sino en algo que se contempla a sí mismo a través de una metáfora que a su vez puede estar basada en otra metáfora, lo cual, sin lugar a dudas, provoca cierta ambigüedad, así como la pérdida de un equilibrio, una estabilidad, que en el caso de un texto escrito puede llevar a la disolución de la figura del autor y a la diseminación de cualquier origen posible.

Ciertamente, el término "diseminación" se presenta como palabra clave a la hora de intentar aferrarse a la idea de origen. Quizá la explicación más técnica de la palabra viene dada de nuevo por Hayles, quien relaciona la diseminación con el término científico "entropía," derivado de un vocablo griego que significa "transformación." Además de proporcionar una detallada explicación del término en clave científica, Hayles lo vincula a la idea de disipación de energía, así como a la división de la unidad original en los distintos elementos que la componen. Diseminar significa originalmente "expander semillas," y esta expansión inevitablemente conduce a la idea de intertextualidad, así como al concepto de hipertexto y al empleo indiscriminado de citas. En su obra Citation and Modernity, Claudette Sartiliot vincula la diseminación textual al uso de referencias tomadas de otras fuentes. Las citas, o las palabras anteriormente usadas por otros, constituyen uno de los actos más peligrosos de rebelión cometidos por los textos que se analizan aquí, calificados como

\footnotetext{
${ }^{1}$ Se ha usado aquí una edición en inglés del Libro de los Muertos, The Egyptian Book of the Dead (Ed. E.A. Wallis Budge, NY: Dover Publications, 1967). Las referencias entre corchetes pertenecen al editor. La cita consiste en la transliteración de los signos jeroglíficos que, por motivos de dificultades técnicas, no han sido incluidos en este trabajo.
} 
textos "vengativos." Podría afirmarse que el empleo de citas es, al igual que el metadiscurso, tan remoto como la propia escritura, y de forma irónica, las citas en la época clásica se convierten en un elemento imprescindible que dota al texto de originalidad. Esta vez, no obstante, Sartiliot se plantea: "is a quotation still a quotation when it has become distorted beyond recognition, when one no longer knows with any certainty whom to attribute it to, if the boundaries between text and intertext, if the notions of closure, paternity, totality have themselves been subverted?" (74). Curiosamente, Sartiliot se cuestiona esta duda en relación con un texto muy particular, la última obra de Joyce, Finnegans Wake, que es definida por la autora como "a network of quotations" (89), donde es frecuente encontrar citas que han sido alteradas, sin hallar por el contrario ni un sólo signo de puntuación, comillas o guiones, que indiquen que se trata de apropiaciones de otras obras o de otros autores. El propio texto se justifica al hablar del mecanismo usado en su composición. La carta, que en Finnegans Wake se erige como imagen recurrente que hace las veces de la obra en su totalidad entendida como creación literaria, justifica la interpretación:

that its page cannot ever have been a penproduct of a man or woman of that period or those parts is only one more unlooked for conclusion leaped at, being tantamount to inferring from the nonpresence of inverted commas (sometimes called quotation marks) on any page that its author was always constitutionally incapable of misapropriating the spoken words of others (108. 30-36).

La ausencia de comillas contribuye a despertar la confusión y la ambigüedad, y de esta manera, citar se convierte en una diseminación, ocultando la figura del autor como también destruyendo cualquier esperanza de encontrar un origen. Por esta razón, Sartiliot no habla de apropiaciones, sino de "missapropriations," citas susceptibles de ser alteradas y traicionar la fuente original. El problema, en cualquier caso, viene dado por el inconveniente de que ya no queda claro cuál es la fuente original o si existe alguna.

Igualmente, como defiende Hayles, esta disipación o expansión viene incrementada por una proliferación de información: a mayor diseminación, más cantidad de información, aunque tal información esté colmada de ruido, de errores y equivocaciones. En la mayoría de los casos, dichos errores son debidos a una falta de eficacia en la transmisión, cuanto más si se trata de una transmisión oral. De nuevo es éste el caso de Finnegans Wake, cuyo argumento principal parece estar basado en el juego recurrente de la narración de la misma historia, la caída o el pecado protagonizado por HCE, y narrada en multitud de ocasiones de manera oral, de forma que se obtiene una lista interminable de versiones distorsionadas de un mismo suceso, en un proceso parecido al célebre juego infantil del teléfono, donde la participación de distintos emisores causan la tergiversación del mensaje original. También la escasa efectividad de la transmisión oral juega un papel determinante en la novela de Charles Palliser titulada Betrayals. Imitando el estilo de las historias detectivescas, la obra está dividida en distintos capítulos que se aluden unos a otros, en los que el lector encuentra diferentes narradores, cada uno de ellos ofreciendo una relación alterada de lo que parece ser la misma historia. Es difícil aclarar lo que realmente sucede o incluso si alguno de estos narradores es fiable. Por ejemplo, uno de los capítulos muestra un narrador que relata sus experiencias tras inscribirse en un seminario sobre estudios culturales y crítica literaria. El seminario es impartido por un profesor, Galvanauskas, que sospechosamente se niega a dar 
sus clases por escrito o a permitir que los asistentes tomen notas, a semejanza de Platón, cuyas enseñanzas son recogidas por escrito por su discípulo Sócrates, debido a la desconfianza del maestro en la escritura. El narrador utiliza una terminología bastante familiar para cualquier lector crítico: "Who is the dominant partner-the text or the reader? And how is this relationship to be established? ... I encountered Galvanauskas's famous essay 'Lo(o)sing the Signifier: Silence, Wordlessness and Desire in Kipling's "The Tongueless Boy"”' (57). Tan sólo unos capítulos más tarde, el lector acoge el punto de vista de otro narrador diferente, quien esta vez refiere lo siguiente:

We met at his place and then went out for a walk and Horatio talked some more about his point on logic and murder and said that the perfect murder is the Sidney Fire (I don't know who he was!) of Desire for everyone involved: reader, writer, detective and murderer ... I have written it down just the way he said it, but I still don't follow it. I must try and find out who Sidney Fire was. Apparently he is or was a friend of someone Horatio seems to know called Gavin -or Galvin? (111).

La función del lector, sin lugar a dudas, es la de archivar información que ya aparecía en capítulos previos, a fin de ser capaz, como lector crítico, de reconstruir lo que evidentemente consiste en un error producido por un fallo en la transmisión oral, a la vez que por un desconocimiento general por parte del segundo narrador de la terminología empleada anteriormente que lo lleva a confundir el "signifier" con un nombre propio.

Dissemination, además, es el título de una colección de ensayos de Jacques Derrida, entre los que incluye el trabajo "The Time Before First." Derrida abre este ensayo con una cita tomada de Through the Looking Glass de Lewis Carroll, el ejemplo perfecto para mostrar la confusión existente entre la realidad y la ficción, así como la entrada a un mundo imaginario, y la pérdida del origen. Derrida declara que: "All oppositions based on the distinction between the original and the derived, the simple and the repeated, the first and the second, etc., lose their pertinence from the moment everything "begins" by following a vestige. I.e. a certain repetition or text" (330). Desde el título mismo, Derrida plantea la imposibilidad de trazar un momento previo a cualquier principio, el origen absoluto.

Fruto de la casualidad, o quizá no, una de las películas más recientes se abre también con una cita de Lewis Carroll, aunque esta vez tomada de Alice in Wonderland. El film en cuestión es The Matrix, que ha sido calificada por algunos críticos y espectadores como una producción comercial típicamente americana. Sin embargo, The Matrix pertenece a una saga de películas iniciada por el ya clásico Blade Runner y continuada por películas como Back to the Future, Terminator (I \& II), 12 Monkeys y The Truman Show, obras donde se da la confusión entre realidad y ficción. Expresada por medio de una mezcla amenazadora de humanos con replicantes, en el caso de Blade Runner, o por medio de una ruptura de la linealidad temporal, como en Back to the Future, Terminator y 12 Monkeys, o incluso mediante un claro ejemplo de metadiscurso en forma de culebrón televisivo, como en The Truman Show, resulta obvio que existe un interés actual en la posibilidad de trazar un ori- 
gen. ${ }^{2}$ Pero quizá la más interesante sea The Matrix, no sólo por ser la más reciente entre las aquí mencionadas, sino porque el título mismo, sugiriendo el origen de todo ser vivo, se aplica al nombre de un programa informático diseñado por criaturas no humanas para engañar nuestra perspectiva sensorial, edificando una realidad virtual. Las repeticiones en este tipo de películas se convierten en un "motif" que deja la sensación de "dejà vu." Asimismo, la expresión "un error en Matrix" ha sido adoptada por el vocabulario popular para referirse a los instantes de bloqueo mental o de "dejà vu" que uno experimenta de vez en cuando.

Es evidente, pues, que existe un interés particular en nuestra cultura por cuestionar aspectos del ser humano que habían sido dados por hechos, asimilados de forma dogmática, como la eficacia del lenguaje, la superioridad de la raza humana sobre el resto de la creación y' también la posibilidad de trazar una arqueología del mundo que nos rodea. El error principal reside en la creencia en el desarrollo lineal de la Historia, en una relación causaefecto, o principio-fin, sin atenerse a las innumerables rupturas de esa estructura lineal o a la influencia que eventos presentes o incluso futuros pueden ejercer sobre hechos pasados. Un ejemplo demasiado simplista pero que sirve de ayuda para ilustrar esta idea es la proliferación de teléfonos móviles. En un momento en que ya no resulta bochornoso recibir una llamada en el supermercado o en la parada de autobús, cuando el móvil se transforma en un elemento cotidiano más como la televisión o la lavadora, lo que antes era un teléfono, simplemente, se bautiza de nuevo con el nombre de "teléfono fijo," sólo porque un artefacto posterior ejerce una influencia sobre algo anterior, modificando nuestra percepción del primer objeto.

Concerniente a la literatura, el lector está acostumbrado a las novelas que siguen un comienzo, un intermedio y un final, sin percibir que esta organización es sólo el producto de un esfuerzo por parte del autor de ordenar los hechos de forma lineal, cuando la vida cotidiana demuestra que todo ocurre de forma contraria, y que dos o varios sucesos pueden tener lugar al mismo tiempo o incluso entrecruzarse. Sin duda alguna, esto ocurre en el episodio "Wandering Rocks" del Ulysses de Joyce, donde el autor irlandés introduce el concepto de paralaje o diferentes perspectivas del mismo objeto, al igual que en La Colmena de Camilo José Cela, donde las vidas de los personajes se entremezclan aunque no se conozcan los unos a los otros, entre muchas otras obras.

Siguiendo esta línea, el crítico Fritz Senn, célebre por su costumbre de plantear cuestiones que, por su propia obviedad escapan a nuestro ojo analítico, estudia en "History as Text in Reverse" el influjo de eventos históricos posteriores sobre hechos acontecidos en el pasado. Es éste el motivo que le lleva a mencionar la contradicción implícita en el uso de las siglas B.C. y A.C. (antes y después de Cristo) aclarando que: "Nothing whatever happened in B.C.; there was no B.C. until C." (770), ejemplo que resulta muy similar al propuesto anteriormente en relación con el uso de la telefonía móvil.

\footnotetext{
${ }^{2}$ Cabe añadir a esta lista una de las últimas adquisiciones cinematográficas, The Blair Witch Project, aunque quizá no merezca tanta atención la cinta en sí, como toda la parafernalia internauta erigida en torno al guión, cuyo objeto principal es crear una mezcla entre lo verídico y lo ficticio.
} 
Quizá sea el momento de considerar las citas literarias como parodias, puesto que una cita es siempre una re-invención del pasado, una re-escritura, de la misma manera que el acto de leer implica una nueva creación, además de un proceso por el que de una forma u otra se está citando. La influencia del presente y el futuro sobre el pasado genera una estructura circular, de la cual Finnegans Wake una vez más constituye el modelo idóneo. La obra es un texto perfectamente circular, en el que no hay un origen ni tampoco un final. Es un tipo de escritura a la que se puede acceder sin tener una entrada definida ni una salida concreta. Una escritura que asimismo otorga de la calidad creativa a la labor de plagio, disipando la distinción entre original y derivado, primario y secundario. Finnegans Wake se rebela como un acto de venganza sobre

that ideal reader suffering from an ideal imsomnia . . . calling unnecessaryattention to errors, omissions, repetitions and misalignments . . . the learning betrayed at almost every line's end: the headstrength (at leasteleven men of thritytwo pallfrycraft) revealed by a constant labour to make a ghimel pass through the eye of an iota (120.13-27).

De forma incuestionable, Finnegans Wake se levanta como cabeza visible del tipo de textos que aquí se discute, ejecutando una venganza en contra de las imposiciones pasadas sobre la figura del autor, una venganza contra los constreñimientos relacionados con la perspectiva lineal de la Historia y de las historias, y una venganza cometida contra la opresión ejercida por la autoridad de las citas en el planteamiento clásico. Pero sobre todo, hay que tener en cuenta el aspecto más relevante y a la vez más traumático, el hecho de que Finnegans Wake representa el modelo perfecto de la venganza más cruel cometida contra nosotros, como lectores y escritores del texto.

\section{OBRAS CITADAS}

Back to the Future. Dir. Robert Zemeckis. Con Michael J. Fox y Christopher Lloyd. Universal Pictures \& Amblin Entertainment, 1985.

Blade Runner. Dir. Ridley Scott. Con Harrisond Ford, Sean Young y Daryl Hannah. Prod. Co. Blade Runner Partnership \& The Ladd Co, 1985.

Carroll, Lewis. Alice in Wonderland \& Through the Looking Glass. Hertfordshire: Wordsworth Editions Ltd., 1993.

Cela, Camilo José. La Colmena. Barcelona: Editorial Laia, 1982.

Derrida, Jacques. "The Time Before First." Dissemination. Chicago: U of Chicago P, 1981.

Escher, M. Relativity. Cornellius Van S. Roosevelt Collection, 1953.

Hayles, Katherine. Chaos Bound. Orderly Disorder in Contemporary Literature and Science. Ithaca \& London: Cornell UP, 1990.

Joyce, James. Finnegans Wake. London: The Penguin Group, P.B. Ltd., 1992.

---. Ulysses. Eds. Hans Walter Gabler, Wolfhard Steppe y Claus Melchior. London: Penguin Books Ltd., 1986. 
Palliser, Charles. Betrayals. London: Jonathan Cape Ltd., 1994.

Said, Edward. Beginnings: Intention and Method. NY: Columbia UP, 1985.

Sartiliot, Claudette. Citation and Modernity: Derrida, Joyce and Brecht. Norman \& London: U of Oklahoma P, 1993.

Senn, Fritz. "History as Text in Reverse." James Joyce Quarterly. 28.4 (1991): 765-76.

Sterne, Laurence. The Life and Opinions of Tristram Shandy. London: The Penguin Group, 1967.

The Terminator y Terminator 2: Judgement Day. Dir. James Cameron. Con Arnold Schwarzenagger y Linda Hamilton. Carolco Pictures Inc., 1984 y 1991.

The Blair Witch Project. Dir. Daniel Myrick y Eduardo Sánchez. Artisan Pictures Inc., 1999.

The Egyptian Book of the Dead. Ed. E.A. Wallis Budge. NY: Dover Publications, 1967.

The Matrix. Dir. Larry y Andy Wachowsky. Con Keanu Reeves, Laurence Fishburne y Carrie-Anne Moss. Warner Bross. Pictures, 1999.

The Truman Show. Dir. Peter Weir. Con Jim Carrey. Paramount Pictures, 1998.

12 Monkeys. Dir. Terry Gilliam. Con Brad Pitt, Bruce Willis y Madeleine Stowe. Universal Pictures, Classico \& Atlas Entertainment, 1995.

Vega, Lope de. "Un Soneto me Manda . . ." Poesía Selecta. Ed. Antonio Carreño. Madrid: Ediciones Cátedra S.A., 1984. 
